\title{
UNIFORM ASYMPTOTIC NORMAL STRUCTURE, THE UNIFORM SEMI-OPIAL PROPERTY AND FIXED POINTS OF ASYMPTOTICALLY REGULAR UNIFORMLY LIPSCHITZIAN SEMIGROUPS. PART I
}

\author{
MONIKA BUDZYŃSKA, TADEUSZ KUCZUMOW AND SIMEON REICH
}

\begin{abstract}
In this paper we introduce the uniform asymptotic normal structure and the uniform semi-Opial properties of Banach spaces. This part is devoted to a study of the spaces with these properties. We also compare them with those spaces which have uniform normal structure and with spaces with $W C S(X)>1$.
\end{abstract}

\section{Introduction}

Normal structure is one of the basic concepts in metric fixed point theory. It was introduced by Brodskii and Milman [6] and applied in Kirk's wellknown fixed point theorem [24]. Asymptotic normal structure appeared for the first time in a paper by Baillon and Schöneberg [4] in which they generalized Kirk's theorem. The semi-Opial property was considered in the context of the fixed point property in product spaces [25]. To study more carefully the geometric structure of Banach spaces Bynum [9] introduced the normal structure coefficient $N(X)$ which was applied by Casini and Maluta [10] to obtain a fixed point theorem for uniformly lipschitzian mappings. This result has been recently improved by Domínguez Benavides [15]. In his paper he used both $N(X)$ and the weakly convergent sequence coefficient $W C S(X)$ [9]. In the first part of the present paper we introduce new geometric coefficients: the asymptotic normal structure and the semi-Opial coefficients. In the second part of our paper we apply them to the fixed point theory of uniformly lipschitzian nonlinear semigroups.

1991 Mathematics Subject Classification. 47H10, 46B20.

Key words and phrases. Uniform asymptotic normal structure, the uniform semi-Opial property.

Received: September 15, 1997. 


\section{The ASymptotic normal StRucture AND the SEMi-Opial COEFFICIENTS}

Let $(X,\|\cdot\|)$ be a Banach space. As we mentioned in the Introduction, Bynum [9] introduced the coefficient $N(X)$ related to normal structure. Namely, he defined $N(X)$ as the biggest constant $k$ such that

$$
k \cdot r(C) \leq \operatorname{diam}(C)
$$

for each nonempty bounded convex set $C \subset X$, where $\operatorname{diam}(C)$ denotes the diameter of $C$ and $r(C)$ is the Chebyshev radius of $C$ with respect to itself, i.e.,

$$
r(C)=\inf _{y \in C} \sup _{x \in C}\|x-y\| .
$$

If $\left\{x_{n}\right\}_{n \geq 1}$ is a bounded sequence in $(X,\|\cdot\|)$ and $\left\{x_{n_{i}}\right\}_{i \geq 1}$ is a subsequence, then we denote by $r_{a}\left(\left\{x_{n_{i}}\right\}_{i \geq 1}\right)$ the asymptotic radius for the norm $\|\cdot\|$ of this subsequence with respect to the set $\overline{\operatorname{conv}}\left(\left\{x_{n}\right\}_{n \geq 1}\right)$ ( the closure in the norm $\|\cdot\|$ of the convex hull of the whole sequence $\left.\left\{x_{n}\right\}_{n \geq 1}\right)$, i.e.,

$$
\begin{gathered}
r_{a}\left(\left\{x_{n_{i}}\right\}_{i \geq 1}\right)= \\
=\inf \left\{r_{a}\left(x,\left\{x_{n_{i}}\right\}_{i \geq 1}\right)=\limsup _{i}\left\|x-x_{n_{i}}\right\|: x \in \overline{\operatorname{conv}}\left(\left\{x_{n}\right\}_{n \geq 1}\right)\right\} .
\end{gathered}
$$

Throughout this paper we will use the following notation:

$$
\alpha_{k}=\operatorname{diam}_{\|\cdot\|}\left(\left\{x_{n}\right\}_{n \geq k}\right), \quad \operatorname{diam}_{a}\left(\left\{x_{n}\right\}\right)=\lim _{k} \alpha_{k}=\alpha .
$$

One can consider (see [9] and [2]) the following weakly convergent sequence coefficient:

$$
W C S(X)=\sup \left\{k: k \cdot r_{a}\left(\left\{x_{n}\right\}\right) \leq \operatorname{diam}\left(\left\{x_{n}\right\}\right)\right.
$$

for every weakly convergent sequence $\left\{x_{n}\right\}$ in $\left.X\right\}=$

$$
=\sup \left\{k: k \cdot \limsup _{n}\left\|x_{n}\right\| \leq \operatorname{diam}\left(\left\{x_{n}\right\}\right)\right.
$$

for every weakly null sequence $\left\{x_{n}\right\}$ in $\left.X\right\}$.

Let us observe that in the above definition of $W C S(X), \operatorname{diam}\left(\left\{x_{n}\right\}\right)$ can be replaced by $\operatorname{diam}_{a}\left(\left\{x_{n}\right\}\right)$ and that our definition is a little different from the one in common use.

We always have

$$
1 \leq N(X) \leq W C S(X),
$$

and for some Banach spaces (see e.g. [15]) the strict inequalities

$$
1<N(X)<W C S(X)
$$

are valid.

Recall that a bounded sequence $\left\{x_{n}\right\}_{n \geq 1}$ with $x_{n}-x_{n+1} \rightarrow 0$ is called asymptotically regular. 
We say that $X$ has asymptotic normal structure (with respect to the weak topology) [4], $A N S$ (respectively, $w-A N S$ ) for short, if for each bounded closed (weakly compact) and convex subset $C$ of $X$ consisting of more than one point and each asymptotically regular sequence $\left\{x_{n}\right\}$ in $C$, there is a point $x \in C$ such that

$$
\liminf _{n}\left\|x-x_{n}\right\|<\operatorname{diam}(C)
$$

(see also $[1,2,7,8,19,20,26,30,36]$ ).

Recall that a Banach space is said to have the semi-Opial (weak semiOpial) property $[8,25], S O(w-S O)$ for short, if for each bounded nonconstant asymptotically regular sequence $\left\{x_{n}\right\}$ (with a weakly compact convex hull), there exists a subsequence $\left\{x_{n_{i}}\right\}$, weakly convergent to $x$, such that

$$
\liminf _{i}\left\|x-x_{n_{i}}\right\|<\operatorname{diam}\left(\left\{x_{n}\right\}\right) \text {. }
$$

Let us observe that in Examples 1 and 5 on page 461 in [25] the authors use, in fact, the weak semi-Opial property. Similarly in Theorem 4 in [25] we can assume that $\left(X_{2},\|\cdot\|\right)$ has the weak semi-Opial property.

A Banach space $X$ is said to satisfy the Opial condition [32] (respectively, the nonstrict Opial condition [22]) if whenever a sequence $\left\{x_{n}\right\}$ in $X$ converges weakly to $x$, then

$$
\begin{gathered}
\liminf _{n}\left\|x-x_{n}\right\|<\liminf _{n}\left\|y-x_{n}\right\| \\
\left(\liminf _{n}\left\|x-x_{n}\right\| \leq \liminf _{n}\left\|y-x_{n}\right\|\right)
\end{gathered}
$$

for every $y \in X \backslash\{x\}$.

For more information about the connections between the above mentioned geometric properties of Banach spaces (and other ones) see [1, 2, 3, 13, 14, 18, $19,20,27,29,33,34,35,37,38,39,40]$.

We now define the asymptotic normal structure coefficient by

$$
\sup \left\{k: k \cdot \inf _{\left\{x_{n_{i}}\right\}_{i \geq 1}} r_{a}\left(\left\{x_{n_{i}}\right\}_{i \geq 1}\right) \leq \operatorname{diam}_{a}\left(\left\{x_{n}\right\}\right)\right.
$$

for each bounded sequence $\left\{x_{n}\right\}_{n \geq 1}$ with $\left.x_{n}-x_{n+1} \rightarrow 0\right\}$.

We denote it by $A N(X)$.

If in the definition of $A N(X)$ we add the condition that the sequence $\left\{x_{n}\right\}_{n \geq 1}$ has a weakly compact $\overline{c o n v}\left(\left\{x_{n}\right\}_{n \geq 1}\right)$, then we get the asymptotic normal structure coefficient with respect to the weak topology, $w-A N(X)$, for short. In other words,

$$
w-A N(X)=\sup \left\{k: k \cdot \inf _{\left\{x_{n_{i}}\right\}_{i \geq 1}} r_{a}\left(\left\{x_{n_{i}}\right\}_{i \geq 1}\right) \leq \operatorname{diam}_{a}\left(\left\{x_{n}\right\}\right)\right.
$$

for each sequence $\left\{x_{n}\right\}_{n \geq 1}$ such that 


$$
\left.\overline{\operatorname{conv}}\left(\left\{x_{n}\right\}_{n \geq 1}\right) \text { is weakly compact and } x_{n}-x_{n+1} \rightarrow 0\right\} \text {. }
$$

The semi-Opial coefficient with respect to the weak topology, $w$-SOC for short, is defined as follows:

$$
w-S O C(X)=\sup \left\{k: k \cdot \inf _{\left\{x_{n_{i}}\right\}_{i \geq 1}, x_{n_{i}} \rightarrow y} r_{a}\left(y,\left\{x_{n_{i}}\right\}_{i \geq 1}\right) \leq \operatorname{diam}_{a}\left(\left\{x_{n}\right\}\right)\right.
$$

for each sequence $\left\{x_{n}\right\}_{n \geq 1}$ such that

$$
\left.\overline{\operatorname{conv}}\left(\left\{x_{n}\right\}_{n \geq 1}\right) \text { is weakly compact and } x_{n}-x_{n+1} \rightarrow 0\right\} \text {. }
$$

If $A N(X)>1$, then we say that $(X,\|\cdot\|)$ has uniform asymptotic normal structure, $U A N$ for short. If $w-A N(X)>1$, then we say that $(X,\|\cdot\|)$ has uniform asymptotic normal structure with respect to the weak topology $(w-U A N)$. Similarly, if $w$-SOC $(X)>1$, then $(X,\|\cdot\|)$ has the uniform semi-Opial property with respect to the weak topology $(w-U S O)$.

Directly from the above definitions we get

$$
\begin{gathered}
1 \leq A N(X) \leq w-A N(X), \\
1 \leq W C S(X) \leq w-S O C(X) \leq w-A N(X) .
\end{gathered}
$$

We do not know if $w$-AN $(X)$ is different from $w$-SOC $(X)$, but we will present an example of a Banach space with $1<W C S(X)<w$-SOC $(X)$ (Example 6.2). There are Banach spaces which have asymptotic normal structure but lack $U A N$, and there are also Banach spaces with $1=A N(X)<$ $w$-AN (X) (Example 6.1).

Proposition 2.1. In the definitions of $w-A N(X)$ and $w-S O C(X)$ we can replace $\operatorname{diam}_{a}\left(\left\{x_{n}\right\}\right)$ by $\operatorname{diam}\left(\left\{x_{n}\right\}\right)$.

Proof. Let us observe that in the above definition every asymptotically regular sequence $\left\{x_{n}\right\}$ can be replaced by $\left\{x_{n}\right\}_{n \geq m}$ with arbitrary $m$. This yields the claimed statement.

Theorem 2.1. If a Banach space $(X,\|\cdot\|)$ has $A N(X)>1$, then it is reflexive.

Proof. It is sufficient to recall the following result of D.P. Milman and V.D. Milman [31]: If a Banach space $(X,\|\cdot\|)$ is not reflexive, then for each $\epsilon>0$ there exists a sequence $\left\{y_{n}\right\}$ with the following properties:

1. $\left\|y_{n}\right\|=1$ for $n=1,2, \ldots$;

2. $1+\epsilon \geq\left\|z_{1 j}-z_{j \omega}\right\| \geq 1-\epsilon$ for each $j=1,2, \ldots$ and for each $z_{1 j} \in$ $\overline{\operatorname{conv}}\left(\left\{y_{n}\right\}_{n=1}^{j}\right)$ and $z_{j \omega} \in \overline{\operatorname{conv}}\left(\left\{y_{n}\right\}_{n=j+1}^{\infty}\right)$;

3. $1-\epsilon \leq\left\|z_{1 j}\right\| \leq 1$ and $1-\epsilon \leq\left\|z_{j \omega}\right\| \leq 1$ for each $z_{1 j} \in \overline{\operatorname{conv}}\left(\left\{y_{n}\right\}_{n=1}^{j}\right)$ and $z_{j \omega} \in \overline{\operatorname{conv}}\left(\{y\}_{n=j+1}^{\infty}\right)$. 
Hence, if a Banach space $(X,\|\cdot\|)$ is not reflexive and $\epsilon>0$, then we can choose elements $y_{n}$ which satisfy the above conditions 1.-3. and next we construct an asymptotically regular sequence $\left\{x_{n}\right\}$ by dividing every segment $\left[y_{n}, y_{n+1}\right]$ into $2^{n}$ equal subsegments and taking their endpoints as subsequent elements of $\left\{x_{n}\right\}$. For this bounded sequence $\left\{x_{n}\right\}_{n \geq 1}$ we have $x_{n}-x_{n+1} \rightarrow 0$ and $\frac{1+\epsilon}{1-\epsilon} \cdot r_{a}\left(y,\left\{x_{n_{i}}\right\}_{i \geq 1}\right) \geq \operatorname{diam}_{a}\left(\left\{x_{n}\right\}\right)$ for each subsequence $\left\{x_{n_{i}}\right\}_{i \geq 1}$ and $y \in \overline{c o n v}\left\{x_{n}\right\}$.

Remark 2.1. The condition $w-A N(X)>1$ implies the weak fixed point property for nonexpansive mappings as a consequence of the Baillon-Schöneberg theorem (see also [7]).

Theorem 2.2. i) If a Banach space $(X,\|\cdot\|)$ has $N(X)>1$, then $w-S O C(X)>1$.

ii) If a Banach space $(X,\|\cdot\|)$ has the nonstrict Opial property, then $w-S O C(X)=w-A N(X)$.

Proof. i) If $N(X)>1$, then $X$ is reflexive [29], and

$$
1<N(X) \leq W C S(X) \leq w-S O C(X)
$$

(see (1) and [33]).

ii) We get this equality directly from the definition of the nonstrict Opial property.

Remark 2.2. There exist $w$-USO spaces without the nonstrict Opial property. For example, $L^{p}([0,2 \pi])$ with $1<p<\infty$ and $p \neq 2$ is such a space. It has uniform normal structure, and thus (see point i) in the above theorem) it is w-USO, but it does not satisfy the nonstrict Opial condition [32].

We finish this section by showing the stability of the uniform asymptotic normal structure and the uniform semi-Opial properties.

Theorem 2.3. Let $\left(X_{1},\|\cdot\|_{1}\right)$ and $\left(X_{2},\|\cdot\|_{2}\right)$ be isomorphic Banach spaces and let $d\left(X_{1}, X_{2}\right)$ be the Banach-Mazur distance between them. Then we have

$$
\begin{gathered}
A N\left(X_{1}\right) \leq d\left(X_{1}, X_{2}\right) \cdot A N\left(X_{2}\right), \\
w-A N\left(X_{1}\right) \leq d\left(X_{1}, X_{2}\right) \cdot w-A N\left(X_{2}\right),
\end{gathered}
$$

and

$$
w-S O C\left(X_{1}\right) \leq d\left(X_{1}, X_{2}\right) \cdot w-S O C\left(X_{2}\right) .
$$

Proof. All the inequalities have similar proofs. For example, we prove the third one:

$$
w-S O C\left(X_{1}\right) \leq d\left(X_{1}, X_{2}\right) \cdot w-S O C\left(X_{2}\right) .
$$

Let $\left\{x_{n}\right\}$ be asymptotically regular in $X_{2}$, and let $\overline{c o n v}\left\{x_{n}\right\}$ be weakly compact. Let $T: X_{2} \rightarrow X_{1}$ be an isomorphism and assume $0<k<w$ $S O C\left(X_{1}\right)$. Then there exists a weakly convergent to $y$ subsequence $\left\{T x_{n_{i}}\right\}$ such that

$$
k r_{a}\left(T^{-1} y,\left\{x_{n_{i}}\right\}_{i \geq 1}\right) \leq k\left\|T^{-1}\right\| r_{a}\left(y,\left\{T x_{n_{i}}\right\}_{i \geq 1}\right)
$$




$$
\leq\left\|T^{-1}\right\| \cdot \operatorname{diam}_{a}\left(\left\{T x_{n}\right\}\right) \leq\left\|T^{-1}\right\| \cdot\|T\| \cdot \operatorname{diam}_{a}\left(\left\{x_{n}\right\}\right) .
$$

Hence we get

$$
\frac{k}{\left\|T^{-1}\right\| \cdot\|T\|} \leq w-S O C\left(X_{2}\right)
$$

which yields the claimed inequality.

Remark 2.3. Theorem 2.3 can be understood as a stability result for the weak fixed point property for nonexpansive mappings. This means that if $w-A N\left(X_{1}\right)>1$ and $d\left(X_{1}, X_{2}\right)<w-A N\left(X_{1}\right)$, then $w-A N\left(X_{2}\right)>1$, and by Remark 2.1 the space $X_{2}$ also has the weak fixed point property for nonexpansive mappings.

\section{Connections Between asymptotically Regular Sequences And PROPERTIES OF BANACH SPACES}

It is natural to ask, when either $A N(X)$ or $w-S O C(X)$ is equal to $\infty$. The following theorem gives the answer.

Theorem 3.1. i) $A N(X)=\infty$ if and only if $(X,\|\cdot\|)$ is finite dimensional. ii) $w-S O C(X)=\infty$ if and only if $(X,\|\cdot\|)$ is a Schur space.

iii) $w-A N(X)=\infty$ if and only if $(X,\|\cdot\|)$ is a Schur space.

Proof. i) The equality $A N(X)=\infty$ is equivalent to the following

$\sup \left\{\inf _{\left\{x_{n_{i}}\right\}_{i \geq 1}} r_{a}\left(\left\{x_{n_{i}}\right\}_{i \geq 1}\right):\left\{x_{n}\right\}_{n \geq 1}\right.$ is bounded and $\left.x_{n}-x_{n+1} \rightarrow 0\right\}=0$

If $X$ is finite dimensional, then the above equality is obvious.

When $X$ is infinite dimensional, then by the Riesz Lemma [12] there exists a sequence $\left\{y_{n}\right\}$ such that

$$
\left\|y_{n}\right\|=1 \quad \text { for } \mathrm{n}=1,2, \ldots
$$

and for $n=1,2, \ldots$

$$
\left\|y-y_{n+1}\right\| \geq 1-\frac{1}{n+1} \quad \text { for } \text { every } y \in \operatorname{lin}\left\{y_{1}, y_{2}, \ldots, y_{n}\right\} \text {. }
$$

Let us observe that

$$
\liminf _{n}\left\|x-y_{n}\right\| \geq 1
$$

for each $x \in \operatorname{lin}\left\{y_{n}\right\}$. Now we construct a new sequence $\left\{x_{n}\right\}$ in the following way. We divide each segment $\left[y_{n}, y_{n+1}\right]$ into $2^{n}$ equal parts and take the endpoints as subsequent elements of $\left\{x_{n}\right\}$. This sequence satisfies $x_{n}-$ $x_{n+1} \rightarrow 0$. We will show that for each $x \in \operatorname{lin}\left\{x_{n}\right\}$ we have

$$
\inf _{\left\{x_{n_{i}}\right\}_{i \geq 1}} r_{a}\left(x,\left\{x_{n_{i}}\right\}_{i \geq 1}\right) \geq \frac{1}{4} .
$$

Indeed, every $x_{n}$ can be written in the following way:

$$
x_{n}=\alpha_{n} y_{k(n)}+\left(1-\alpha_{n}\right) y_{k(n)+1} \text {, }
$$


where $0 \leq \alpha_{n} \leq 1$. If we choose any subsequence $\left\{x_{n_{i}}\right\}_{i>1}$, then without loss of generality we may assume that $\alpha_{n_{i}} \rightarrow \alpha$. It is obvious that $k(n) \rightarrow \infty$ and therefore $k\left(n_{i}\right) \rightarrow \infty$ too.

First we claim that for $0 \leq \alpha \leq \frac{3}{4}$ and for each $x \in \operatorname{lin}\left\{x_{n}\right\}=\operatorname{lin}\left\{y_{n}\right\}$ we have

$$
\liminf _{i}\left\|x-x_{n_{i}}\right\| \geq \frac{1}{4} .
$$

Indeed, for such an $\alpha$ we get

$$
\begin{aligned}
\liminf _{i}\left\|x-x_{n_{i}}\right\| & =\liminf _{i}\left\|x-\alpha_{n_{i}} y_{k\left(n_{i}\right)}-\left(1-\alpha_{n_{i}}\right) y_{k\left(n_{i}\right)+1}\right\| \\
& \geq \lim _{i}\left(1-\alpha_{n_{i}}\right)\left(1-\frac{1}{k\left(n_{i}\right)+1}\right)=1-\alpha \geq \frac{1}{4} .
\end{aligned}
$$

Next we obtain

$$
\begin{aligned}
\liminf _{i}\left\|x-x_{n_{i}}\right\| & =\liminf _{i}\left\|x-\alpha_{n_{i}} y_{k\left(n_{i}\right)}-\left(1-\alpha_{n_{i}}\right) y_{k\left(n_{i}\right)+1}\right\| \\
& \geq \liminf _{i}\left\|x-\alpha_{n_{i}} y_{k\left(n_{i}\right)}\right\|-\lim _{i}\left\|\left(1-\alpha_{n_{i}}\right) y_{k\left(n_{i}\right)+1}\right\| \\
& \geq \lim _{i} \alpha_{n_{i}}\left(1-\frac{1}{k\left(n_{i}\right)}\right)-\lim _{i}\left(1-\alpha_{n_{i}}\right)=2 \alpha-1 \geq \frac{1}{2}
\end{aligned}
$$

for $\frac{3}{4} \leq \alpha \leq 1$ and for each $x \in \operatorname{lin}\left\{x_{n}\right\}$.

Hence (3) and (4) imply that the inequality (2) is valid and therefore

$\sup \left\{\inf _{\left\{x_{n_{i}}\right\}_{n \geq 1}} r_{a}\left(\left\{x_{n_{i}}\right\}_{i \geq 1}\right):\left\{x_{n}\right\}_{n \geq 1}\right.$ is bounded and $\left.x_{n}-x_{n+1} \rightarrow 0\right\}$ $\geq \frac{1}{4}$.

This means that $A N(X)<\infty$.

ii) If $(X,\|\cdot\|)$ is a Schur space [12], then the following equality $\sup \left\{\inf \left\{r_{a}\left(y,\left\{x_{n_{i}}\right\}_{i \geq 1}\right):\left\{x_{n_{i}}\right\}_{i \geq 1}\right.\right.$ is weakly convergent and $\left.y=w-\lim _{i} x_{n_{i}}\right\}:\left\{x_{n}\right\}_{n \geq 1}$ with a weakly compact $\overline{\operatorname{conv}}\left(\left\{x_{n}\right\}_{n \geq 1}\right)$ and $\left.x_{n}-x_{n+1} \rightarrow 0\right\}=0$

is obvious.

Let us assume that $(X,\|\cdot\|)$ is not a Schur space. We will show $\sup \left\{\inf \left\{r_{a}\left(y,\left\{x_{n_{i}}\right\}_{i \geq 1}\right):\left\{x_{n_{i}}\right\}_{i \geq 1}\right.\right.$ is weakly convergent and $\left.y=w-\lim _{i} x_{n_{i}}\right\}:\left\{x_{n}\right\}_{n \geq 1}$ with a weakly compact $\overline{\operatorname{conv}}\left(\left\{x_{n}\right\}_{n \geq 1}\right)$ and $\left.x_{n}-x_{n+1} \rightarrow 0\right\} \geq \frac{1}{8}$. 
In $X$ there exists a weakly null sequence $\left\{y_{n}\right\}$ with $\left\|y_{n}\right\|=1, n=1,2, \ldots$. Therefore, we can choose a subsequence $\left\{y_{n_{k}}\right\}$ such that for every $y \in$ $\left[y_{n_{k}}, y_{n_{k+1}}\right]$ we have $\|y\| \geq \frac{1}{8}$. Indeed, we take $y_{n_{1}}=y_{0}$ and next if we have chosen $y_{n_{1}}, \ldots, y_{n_{k}}$, then we take $n_{k+1}>n_{k}$ so large that

$$
\left\|(1-\alpha) y_{n_{k}}+\alpha y_{n_{k+1}}\right\| \geq \frac{1}{8}
$$

for every $0 \leq \alpha \leq \frac{3}{4}$. This is possible because by the lower semicontinuity of $\|\cdot\|$ with respect to the weak topology we get

$$
\liminf _{n}\left\|(1-\alpha) y_{n_{k}}+\alpha y_{n}\right\| \geq(1-\alpha)\left\|y_{n_{k}}\right\| \geq \frac{1}{4} .
$$

Now for this $y_{n_{k+1}}$ and each $\frac{3}{4} \leq \alpha \leq 1$ we also have

$$
\left\|(1-\alpha) y_{n_{k}}+\alpha y_{n_{k+1}}\right\| \geq\left\|\alpha y_{n_{k+1}}\right\|-\left\|(1-\alpha) y_{n_{k}}\right\|=2 \alpha-1 \geq \frac{1}{2} .
$$

Now we construct an asymptotically regular sequence $\left\{x_{n}\right\}$ by dividing every segment $\left[y_{n_{k}}, y_{n_{k+1}}\right]$ into $2^{n}$ equal parts and then taking the endpoints as subsequent elements of $\left\{x_{n}\right\}$. It is obvious that

$$
\inf _{\left\{x_{n_{i}}\right\}_{i \geq 1}} r_{a}\left(0,\left\{x_{n_{i}}\right\}_{i \geq 1}\right) \geq \frac{1}{8}
$$

and the proof is complete.

iii) Assume that $X$ is not Schur. We will show that

$$
\begin{aligned}
& \sup \left\{\inf _{\left\{x_{n_{i}}\right\}_{i \geq 1}} r_{a}\left(\left\{x_{n_{i}}\right\}_{i \geq 1}\right):\left\{x_{n}\right\}_{n \geq 1}\right. \text { with a weakly compact } \\
& \left.\overline{\operatorname{conv}}\left(\left\{x_{n}\right\}_{n \geq 1}\right) \text { and } x_{n}-x_{n+1} \rightarrow 0\right\}>0 .
\end{aligned}
$$

We use the asymptotically regular sequence $\left\{x_{n}\right\}$ constructed in the proof of ii). We know that $\left\|x_{n}\right\| \geq \frac{1}{8}$ for each $n$ and that $w$-lim $x_{n}=0$. Now we prove that

$$
\liminf _{n}\left\|x-x_{n}\right\| \geq \frac{1}{16}
$$

for every $x \in X$. Indeed, if $\|x\| \geq \frac{1}{16}$, then by the lower semicontinuity of $\|\cdot\|$ with respect to the weak topology we get

$$
\liminf _{n}\left\|x-x_{n}\right\| \geq\|x\| \geq \frac{1}{16} .
$$

On the other hand for $\|x\| \leq \frac{1}{16}$ we obtain

$$
\liminf _{n}\left\|x-x_{n}\right\| \geq \liminf _{n}\left(\left\|x_{n}\right\|-\|x\|\right) \geq \frac{1}{16}
$$

and this completes the proof.

We end this section with a characterization of reflexive spaces by asymptotically regular sequences. 
Theorem 3.2. A Banach space $(X,\|\cdot\|)$ is reflexive if and only if every asymptotically regular sequence has a weakly convergent subsequence.

Proof. It is known [11] that in reflexive spaces each bounded sequence has a weakly convergent subsequence.

Let us now assume that in the Banach space $(X,\|\cdot\|)$ every asymptotically regular sequence has a weakly convergent subsequence. To get the reflexivity of $(X,\|\cdot\|)$ it is sufficient to prove $([11])$ that each decreasing sequence $\left\{C_{n}\right\}$ of nonempty, bounded, closed and convex sets has a nonempty intersection. Without loss of generality we can assume that $\operatorname{diam}\left(C_{n}\right)>0$ for each $n$. Now we choose $y_{n}$ from each set $C_{n}$ and next we construct an asymptotically regular sequence $\left\{x_{n}\right\}$ by dividing every segment $\left[y_{n}, y_{n+1}\right]$ into $2^{n}$ equal parts and the taking the endpoints as subsequent elements of $\left\{x_{n}\right\}$. This sequence contains a weakly convergent subsequence $\left\{x_{n_{i}}\right\}$. Its weak limit is a common element of $C_{n}$ for $n=1,2, \ldots$.

Remark 3.1. A proof similar to the above one was used in [8] to prove that every Banach space with the $S O$ property is reflexive.

\section{On The 3-SPACE PROBlem}

In this section we consider the following problem: When can the uniform asymptotic normal structure property or the uniform semi-Opial property be extended from a subspace to the whole space? Two slightly different approaches to the solution of this problem will be demonstrated in the following theorems.

Theorem 4.1. Suppose that $X=W \oplus Z$, where $W$ is a closed subspace of $X, Z$ is a Schur space, and the projection onto $W$ has norm 1. Then we have $w$-SOC $(X)=w-S O C(W)$.

Proof. Suppose $\left\{x_{n}\right\}=\left\{w_{n}+z_{n}\right\}$ is an asymptotically regular sequence, $w_{n} \in W, z_{n} \in Z$ for $n=1,2, \ldots$ and $\overline{c o n v}\left\{x_{n}\right\}$ is weakly compact. For each $k<w-S O C(W)$ we find a subsequence $\left\{x_{n_{i}}\right\}$ such that

$$
x_{n_{i}}=w_{n_{i}}+z_{n_{i}} \rightarrow w+z, \quad w \in W, z \in Z
$$

and

$$
k \lim _{i}\left\|w_{n_{i}}-w\right\| \leq \operatorname{diam}_{a}\left\{w_{n}\right\} .
$$

Then we have $w+z \in \overline{\operatorname{conv}}\left\{x_{n}\right\}, z_{n_{i}} \rightarrow z$ and

$$
k \lim _{i}\left\|w_{n_{i}}+z_{n_{i}}-w-z\right\|=k \lim _{i}\left\|w_{n_{i}}-w\right\| \leq \operatorname{diam}_{a}\left\{w_{n}\right\} .
$$

Now, since the projection on $W$ is of norm 1 , we obtain

$$
k \lim _{i}\left\|w_{n_{i}}+z_{n_{i}}-w-z\right\| \leq \operatorname{diam}_{a}\left\{w_{n}\right\} \leq \operatorname{diam}_{a}\left\{x_{n}\right\}
$$

and therefore $w$-SOC $(X)=w$-SOC $(W)$.

Now we consider the Cartesian product of two spaces. 
Theorem 4.2. Let $\left(X_{1},\|\cdot\|_{1}\right)$ and $\left(X_{2},\|\cdot\|_{2}\right)$ be Banach spaces. If $\left(X_{1},\|\cdot\|_{1}\right)$ is $w$-USO and $\left(X_{2},\|\cdot\|_{2}\right)$ has $W C S\left(X_{2}\right)>1$, then $X_{1} \times X_{2}$ equipped with the $l_{p}$-norm $\|\cdot\|=\left(\|\cdot\|_{1}^{p}+\|\cdot\|_{2}^{p}\right)^{\frac{1}{p}}(1 \leq p<\infty)$ is also $w-U S O$.

Proof. Let $0<\theta<1$ be such that $\frac{1}{\theta}<\min \left(w-S O C\left(X_{1}\right), W C S\left(X_{2}\right)\right)$. Let us take an arbitrary asymptotically regular sequence $\left\{x_{n}\right\}=\left\{\left(x_{1 n}, x_{2 n}\right)\right\}$ in $\left(X_{1} \times X_{2},\|\cdot\|\right)$ with a weakly compact $\overline{c o n v}\left\{x_{n}\right\}$. Then $\left\{x_{1 n}\right\}$ is also asymptotically regular in $\left(X_{1},\|\cdot\|_{1}\right)$ and we can choose a subsequence $\left\{x_{n_{i}}\right\}$ such that $\left\{x_{n_{i}}\right\}$ tends weakly to $\left(x_{1}, x_{2}\right)$ (see $\left.[13,17,33,40]\right)$ and

$$
\begin{aligned}
d & =\operatorname{diam}_{a}\left\{x_{n}\right\} \\
& \geq \operatorname{diam}_{a}\left\{x_{n_{i}}\right\}=\lim _{\substack{i, k \rightarrow \infty \\
i \neq k}}\left\|x_{n_{i}}-x_{n_{k}}\right\|=\lim _{i \rightarrow \infty} \lim _{k \rightarrow \infty}\left\|x_{n_{i}}-x_{n_{k}}\right\|, \\
r & =\lim _{i \rightarrow \infty}\left\|x_{n_{i}}-x\right\|, \\
d_{1} & =\operatorname{diam}_{a}\left\{x_{1 n}\right\} \\
\geq \bar{d}_{1} & =\operatorname{diam}_{a}\left\{x_{1 n_{i}}\right\}=\lim _{\substack{i, k \rightarrow \infty \\
i \neq k}}\left\|x_{1 n_{i}}-x_{1 n_{k}}\right\|_{1}=\lim _{i \rightarrow \infty} \lim _{k \rightarrow \infty}\left\|x_{1 n_{i}}-x_{1 n_{k}}\right\|_{1}, \\
r_{1} & =\lim _{i \rightarrow \infty}\left\|x_{1 n_{i}}-x_{1}\right\|_{1} \leq \theta d_{1}, \\
\bar{d}_{2} & =\operatorname{diam}_{a}\left\{x_{2 n_{i}}\right\}=\lim _{\substack{i, k \rightarrow \infty \\
i \neq k}}\left\|x_{2 n_{i}}-x_{2 n_{k}}\right\|_{2}=\lim _{i \rightarrow \infty} \lim _{k \rightarrow \infty}\left\|x_{2 n_{i}}-x_{2 n_{k}}\right\|_{2}, \\
r_{2} & =\lim _{i \rightarrow \infty}\left\|x_{2 n_{i}}-x_{2}\right\|_{2} .
\end{aligned}
$$

Let us observe that

$$
\begin{gathered}
d_{1} \leq d, \\
r^{p}=r_{1}^{p}+r_{2}^{p} \leq \bar{d}_{1}^{p}+\bar{d}_{2}^{p} \leq d^{p},
\end{gathered}
$$

$$
r_{1} \leq \theta d_{1} \leq \theta d
$$

and

$$
r_{2} \leq \theta \bar{d}_{2}
$$

Now we have to consider two possibilities: either

$$
r_{1}^{p}+\bar{d}_{2}^{p} \leq \frac{1+3 \theta^{p}}{4} d^{p}
$$

or

$$
r_{1}^{p}+\bar{d}_{2}^{p} \geq \frac{1+3 \theta^{p}}{4} d^{p} .
$$

For the first possibility we obtain

$$
r^{p}=r_{1}^{p}+r_{2}^{p} \leq r_{1}^{p}+\bar{d}_{2}^{p} \leq \frac{1+3 \theta^{p}}{4} d^{p} .
$$

For the second possibility we have

$$
\bar{d}_{2}^{p} \geq \frac{1+3 \theta^{p}}{4} d^{p}-r_{1}^{p} \geq \frac{1+3 \theta^{p}}{4} d^{p}-\theta^{p} d^{p}=\frac{1-\theta^{p}}{4} d^{p}
$$


by (5) and therefore we get

$$
\begin{aligned}
r^{p} & =r_{1}^{p}+r_{2}^{p} \leq r_{1}^{p}+\theta^{p} \bar{d}_{2}^{p} \\
& \leq \bar{d}_{1}^{p}+\bar{d}_{2}^{p}-\left(1-\theta^{p}\right) \bar{d}_{2}^{p} \leq d^{p}-\frac{\left(1-\theta^{p}\right)^{2}}{4} d^{p} \\
& =\left[1-\frac{\left(1-\theta^{p}\right)^{2}}{4}\right] d^{p} .
\end{aligned}
$$

Finally, inequalities (6) and (7) imply

$$
\begin{aligned}
r & \leq \max \left\{\left(\frac{1+3 \theta^{p}}{4}\right)^{\frac{1}{p}},\left[1-\frac{\left(1-\theta^{p}\right)^{2}}{4}\right]^{\frac{1}{p}}\right\} d \\
& =\left[1-\frac{\left(1-\theta^{p}\right)^{2}}{4}\right]^{\frac{1}{p}} d .
\end{aligned}
$$

This completes the proof.

\section{The SPACE $X_{\beta}^{p}$ AND ITS $w$-SOC}

In this section we give an example of a space with $N(X)<A N(X)<$ $w$-SOC $(X)$. To this end, let us consider $l^{p}$ with the norm

$$
\|x\|=\max \left\{\|x\|_{\infty}, \frac{\|x\|_{p}}{\beta}\right\},
$$

where $p>1,1<\beta<+\infty,\|x\|_{\infty}=\max \{|x(j)|: j=1,2, \ldots\}$ and $\|x\|_{p}=\left(\sum_{j=1}^{\infty}|x(j)|^{p}\right)^{\frac{1}{p}}$. We denote this space by $X_{\beta}^{p}$. The space $X_{\beta}^{2}$ was introduced by R.C. James [5]. This is essentially the space which has been discussed in various places in the literature, e.g., $[1,2,4,5,7,8,10,15,16,19,20$, $21,22,23,25,26,28,39]$.

For the convenience of the reader we recall the notations from Section 2 . If $\left\{x_{n}\right\}_{n \geq 1}$ is a bounded sequence in $\left(l^{p},\|\cdot\|\right)$ and $\left\{x_{n_{i}}\right\}_{i \geq 1}$ is a subsequence, then $r_{a}\left(\left\{x_{n_{i}}\right\}_{n \geq 1}\right)$ denote the asymptotic radius of this sequence with respect to the set $\overline{\operatorname{conv}}\left(\left\{x_{n}\right\}_{n \geq 1}\right)$ in the norm $\|\cdot\|$. We also have

$$
\alpha_{k}=\operatorname{diam}_{\|\cdot\|}\left(\left\{x_{n}\right\}_{n \geq k}\right) \quad \text { and } \operatorname{diam}_{a}\left(\left\{x_{n}\right\}\right)=\lim _{k} \alpha_{k}=\alpha .
$$

Let us observe that for each $n \in \mathbb{N}$ and for each $y \in C$ there exists an index $j_{n, y}$

(we fix it here for every pair $n, y$ ) such that

$$
\left\|x_{n}-y\right\|_{\infty}=\left|x_{n}\left(j_{n, y}\right)-y\left(j_{n, y}\right)\right|
$$

$\left(x_{n}=\left(x_{n}(j)\right)_{j \geq 1}\right.$ and $\left.y=(y(j))_{j \geq 1}\right)$.

The space $X_{\beta}^{p}$ has the nonstrict Opial property [22]. 
Theorem 5.1. If a sequence $\left\{x_{n}\right\}_{n \geq 1}$ is bounded and $x_{n}-x_{n+1} \rightarrow 0$, then

$$
\begin{aligned}
& \inf _{x \in \operatorname{conv}}\left(\left\{x_{n}\right\}_{n \geq 1}\right) \\
& =\inf _{\left\{x_{n_{i}}\right\}}\left[r_{a}\left(\left\{x_{n_{i}}\right\}_{i \geq 1}\right)\right] \\
& \leq \min \left[1, \max \left(2^{-\frac{1}{p}}, \frac{\beta}{4^{\frac{1}{p}}}\right)\right] \cdot \operatorname{diam}_{a}\left(\left\{x_{n}\right\}\right)
\end{aligned}
$$

and this constant is the best possible. Therefore

$$
w-S O C\left(X_{\beta}^{p}\right)=\max \left[1, \min \left(2^{\frac{1}{p}}, \frac{4^{\frac{1}{p}}}{\beta}\right)\right] .
$$

Proof. We begin our proof in the case $1<\beta<4^{\frac{1}{p}}$.

Let

$$
C=\overline{\operatorname{conv}}\left(\left\{x_{n}\right\}_{n \geq 1}\right) \quad \text { and } \quad C_{k}=\overline{\operatorname{conv}}\left(\left\{x_{n}\right\}_{n \geq k}\right), \quad k=1,2, \ldots
$$

Clearly $\operatorname{diam} C_{k}=\alpha_{k}$. Let us observe the following fact. For every subsequence $\left\{x_{n_{i}}\right\}$ which is weakly convergent to $y$ we have $([2,39])$

$$
\begin{aligned}
\liminf _{i}\left\|y-x_{n_{i}}\right\|_{p} & \leq \limsup _{i}\left\|y-x_{n_{i}}\right\|_{p} \leq \frac{1}{2^{\frac{1}{p}}} \operatorname{diam}_{a,\|\cdot\|_{p}}\left(\left\{x_{n_{i}}\right\}\right) \\
& \leq \lim _{k} \frac{1}{2^{\frac{1}{p}}} \operatorname{diam}_{\|\cdot\|_{p}} C_{k} \leq \lim _{k} \frac{\beta}{2^{\frac{1}{p}}} \operatorname{diam}_{\|\cdot\|} C_{k}=\frac{\beta}{2^{\frac{1}{p}}} \alpha .
\end{aligned}
$$

Next choosing in an arbitrary way a subsequence $\left\{x_{n_{i_{l}}}\right\}$ such that

$$
\liminf _{i}\left\|y-x_{n_{i}}\right\|_{\infty}=\lim _{l}\left\|y-x_{n_{i_{l}}}\right\|_{\infty}
$$

we get

$$
\begin{aligned}
\liminf _{i}\left\|y-x_{n_{i}}\right\| & \leq \liminf _{l} \max \left\{\left\|y-x_{n_{i_{l}}}\right\|_{\infty}, \frac{\left\|y-x_{n_{i_{l}}}\right\|_{p}}{\beta}\right\} \\
& \leq \max \left\{\lim _{l}\left\|y-x_{n_{i_{l}}}\right\|_{\infty}, \limsup _{l} \frac{\left\|y-x_{n_{i_{l}}}\right\|_{p}}{\beta}\right\} \\
& \leq \max \left\{\lim _{l}\left\|y-x_{n_{i_{l}}}\right\|_{\infty}, \frac{\alpha}{2^{\frac{1}{p}}}\right\} \\
& =\max \left\{\liminf _{i}\left\|y-x_{n_{i}}\right\|_{\infty}, \frac{\alpha}{2^{\frac{1}{p}}}\right\} .
\end{aligned}
$$


Now we can begin the proof of our inequality (9). For $1<\beta<4^{\frac{1}{p}}$ it reduces to

$$
\inf _{x \in \overline{c o n v}\left(\left\{x_{n}\right\}_{n \geq 1}\right)}\left(\liminf _{n}\left\|x_{n}-x\right\|\right) \leq \max \left(2^{-\frac{1}{p}}, \frac{\beta}{4^{\frac{1}{p}}}\right) \cdot \operatorname{diam}_{a}\left(\left\{x_{n}\right\}\right) .
$$

Without loss of generality we can assume that $\alpha>0$, and this implies that for each $k$ we have $\alpha_{k} \geq \alpha>0$. Suppose that for some asymptotically regular sequence $\left\{x_{n}\right\}$ and for some $t$ with $\max \left(\frac{1}{2^{\frac{1}{p}}}, \frac{\beta}{4 \frac{1}{p}}\right)<t<1$ the following inequality is valid:

$$
\inf _{x \in C}\left(\liminf _{n}\left\|x_{n}-x\right\|\right)>t \alpha .
$$

We will try to reach a contradiction. For our $t$ there exists $\epsilon>0$ such that the inequalities

$$
\epsilon<t \alpha \quad \text { and } \quad \frac{\beta^{p}}{2} \alpha^{p}+\epsilon<2(t \alpha-\epsilon)^{p}
$$

are valid.

Let us take an arbitrary subsequence $\left\{x_{n_{i}}\right\}$ which converges weakly to some $y$. Directly from the definition of the norm $\|\cdot\|$, by (11) and by $t>\frac{1}{2^{\frac{1}{p}}}$, we have

$$
\begin{aligned}
t \alpha & <\liminf _{n}\left\|x_{n}-y\right\| \leq \liminf _{i}\left\|x_{n_{i}}-y\right\| \\
& \leq \max \left\{\liminf _{i}\left\|x_{n_{i}}-y\right\|_{\infty}, \frac{\alpha}{2^{\frac{1}{p}}}\right\}=\liminf _{i}\left\|x_{n_{i}}-y\right\|_{\infty} \\
& \leq \liminf _{i} \max \left\{\left\|x_{n_{i}}-y\right\|_{\infty}, \frac{\left\|x_{n_{i}}-y\right\|_{p}}{\beta}\right\}=\liminf _{i}\left\|x_{n_{i}}-y\right\|,
\end{aligned}
$$

which implies

$$
\liminf _{i}\left\|x_{n_{i}}-y\right\|_{\infty}=\liminf _{i}\left\|x_{n_{i}}-y\right\|>t \alpha .
$$

By formulas (8) and (14) and because $\left\{x_{n_{i}}\right\}$ tends weakly to $y$ we get

$$
\lim _{i} j_{n_{i}, y}=+\infty
$$

Using $(8,14,15)$ and $\lim _{n}\left\|x_{n}-x_{n+1}\right\|=0$ we can find $\widetilde{n}$ and $\widetilde{i}, \widetilde{i} \geq \widetilde{n}$, such that for $n \geq \widetilde{n}$ and $i \geq \widetilde{i}$ we have

$$
\begin{gathered}
\left\|x_{n}-x_{n+1}\right\|_{\infty} \leq \frac{\epsilon}{3} \\
\left\|x_{n_{i}}-y\right\|_{\infty}=\left|x_{n_{i}}\left(j_{n_{i}, y}\right)-y\left(j_{n_{i}, y}\right)\right|>t \alpha,
\end{gathered}
$$

and

$$
|y(j)| \leq \frac{\epsilon}{3}
$$


for each $j \geq \min _{i \geq \widetilde{i}} j_{n_{i}, y}$. Therefore (17) and (18) yield

$$
\left\|x_{n_{i}}\right\| \geq\left\|x_{n_{i}}\right\|_{\infty} \geq\left|x_{n_{i}}\left(j_{n_{i}, y}\right)\right| \geq t \alpha-\frac{\epsilon}{3}
$$

for $i \geq \widetilde{i}$.

Now let us return to the sequence $\left\{x_{n}\right\}$ and set

$$
j_{n}= \begin{cases}\max \left\{j:\left|x_{n}(j)\right| \geq t \alpha-\frac{\epsilon}{3}\right\} & \text { if there exists } j \\ \max \left\{j:\left|x_{n}(j)\right|=\left\|x_{n}\right\|_{\infty}\right\} & \text { such that }\left|x_{n}(j)\right| \geq t \alpha-\frac{\epsilon}{3},\end{cases}
$$

We claim that

$$
\lim _{n} j_{n}=+\infty
$$

If this were false, then there would exist a subsequence $\left\{n_{i}\right\}$ with a bounded $\left\{j_{n_{i}}\right\}$. We could then choose a subsequence $\left\{x_{n_{i_{l}}}\right\}$ which tends weakly to its weak limit $y$. In this case (see formulas (19) and (20)) we have

$$
j_{n_{i_{l}}, y} \leq j_{n_{i_{l}}}
$$

for all $l$ greater than or equal to some $\tilde{l}$ and therefore (see (15)) $\lim _{l} j_{n_{i_{l}}}=$ $+\infty$ which contradicts our assumption.

Since $\lim _{n} j_{n}=+\infty$ (see $\left.(21)\right)$, there exists a subsequence $\left\{n_{i}\right\}$ such that $\left\{x_{n_{i}}\right\}$ converges weakly to some $y$ and

$$
j_{n_{i}}<j_{n_{i}+1}
$$

for $i=1,2, \ldots$. Since $\left\|x_{n}-x_{n+1}\right\| \rightarrow 0$ we get $x_{n_{i}+1} \rightarrow y$ and therefore for $i \geq \widetilde{i}$ (see formulas $(16,17,18,19,20)$ ) we get

$$
\begin{gathered}
\left|x_{n_{i}+1}\left(j_{n_{i}+1, y}\right)-y\left(j_{n_{i}+1, y}\right)\right|=\left\|x_{n_{i}+1}-y\right\|_{\infty} \\
\geq\left\|x_{n_{i}}-y\right\|_{\infty}-\left\|x_{n_{i}}-x_{n_{i}+1}\right\|_{\infty} \geq t \alpha-\frac{\epsilon}{3}, \\
\left|x_{n_{i}+1}\left(j_{n_{i}, y}\right)-y\left(j_{n_{i}, y}\right)\right| \\
\geq\left|x_{n_{i}}\left(j_{n_{i}, y}\right)-y\left(j_{n_{i}, y}\right)\right|-\left|x_{n_{i}}\left(j_{n_{i}, y}\right)-x_{n_{i}+1}\left(j_{n_{i}, y}\right)\right| \\
\geq\left\|x_{n_{i}}-y\right\|_{\infty}-\left\|x_{n_{i}}-x_{n_{i}+1}\right\|_{\infty} \geq t \alpha-\frac{\epsilon}{3},
\end{gathered}
$$

and

$$
\begin{aligned}
& \left|x_{n_{i}+1}\left(j_{n_{i}+1}\right)-y\left(j_{n_{i}+1}\right)\right| \geq\left|x_{n_{i}+1}\left(j_{n_{i}+1}\right)\right|-\left|y\left(j_{n_{i}+1}\right)\right| \\
& \geq \min \left\{t \alpha-\frac{\epsilon}{3},\left\|x_{n_{i}+1}\right\|_{\infty}\right\}-\left|y\left(j_{n_{i}+1}\right)\right| \\
& \geq \min \left\{t \alpha-\frac{\epsilon}{3},\left\|x_{n_{i}}\right\|_{\infty}-\left\|x_{n_{i}}-x_{n_{i}+1}\right\|_{\infty}\right\}-\left|y\left(j_{n_{i}+1}\right)\right| \\
& \geq t \alpha-\frac{2 \epsilon}{3}-\frac{\epsilon}{3}=t \alpha-\epsilon .
\end{aligned}
$$

We will now show that there exists $\widetilde{\widetilde{i}}>\widetilde{i}$ such that for $i \geq \widetilde{\widetilde{i}}$ we have $j_{n_{i}+1, y}=j_{n_{i}, y}$. 
Indeed, let us take $\widetilde{\widetilde{i}}>\widetilde{i}$ such that

$$
\left\|y-x_{n_{i}+1}\right\|_{p}^{p} \leq \frac{\beta^{p}}{2} \alpha^{p}+\epsilon
$$

is satisfied for $i \geq \widetilde{\widetilde{i}}$ (this is possible by (10)). If $j_{n_{i}+1, y} \neq j_{n_{i}, y}$, then by $(13,23,24)$ we would obtain

$$
\begin{aligned}
2(t \alpha-\epsilon)^{p} & \leq\left|x_{n_{i}+1}\left(j_{n_{i}, y}\right)-y\left(j_{n_{i}, y}\right)\right|^{p}+\left|x_{n_{i}+1}\left(j_{n_{i}+1, y}\right)-y\left(j_{n_{i}+1, y}\right)\right|^{p} \\
& \leq\left\|x_{n_{i}+1}-y\right\|_{p}^{p} \leq \frac{\beta^{p}}{2} \alpha^{p}+\epsilon<2(t \alpha-\epsilon)^{p} .
\end{aligned}
$$

But this is impossible.

Therefore for every $i \geq \widetilde{\widetilde{i}}$ we have

$$
j_{n_{i}, y}=j_{n_{i}+1, y}
$$

Next by $(16,22,27)$ for $i \geq \widetilde{\widetilde{i}}$ we have

$$
j_{n_{i}+1, y}=j_{n_{i}, y} \leq j_{n_{i}}<j_{n_{i}+1} \text { and }\left\|x_{n_{i}}-x_{n_{i}+1}\right\|_{\infty} \leq \frac{\epsilon}{3} .
$$

Hence by $(13,23,25,26)$ we get the following contradiction

$$
\begin{aligned}
2(t \alpha-\epsilon)^{p} & \leq\left|x_{n_{i}+1}\left(j_{n_{i}+1, y}\right)-y\left(j_{n_{i}+1, y}\right)\right|^{p}+\left|x_{n_{i}+1}\left(j_{n_{i}+1}\right)-y\left(j_{n_{i}+1}\right)\right|^{p} \\
& \leq\left\|x_{n_{i}+1}-y\right\|_{p}^{p} \leq \frac{\beta^{p}}{2} \alpha^{p}+\epsilon<2(t \alpha-\epsilon)^{p} .
\end{aligned}
$$

Thus the sequence $\left\{j_{n_{i}}\right\}$ cannot be strictly increasing, contrary to (22).

Hence the inequality (12) is false and therefore the claimed inequality

$$
\inf _{x \in C}\left(\liminf _{n}\left\|x_{n}-x\right\|\right) \leq t \alpha
$$

is valid for arbitrary $t$ satisfying $\max \left(\frac{1}{2^{\frac{1}{p}}}, \frac{\beta}{4^{\frac{1}{p}}}\right)<t<1$. We conclude that

$$
w-S O C\left(X_{\beta}^{p}\right) \geq \max \left(2^{\frac{1}{p}}, \frac{4^{\frac{1}{p}}}{\beta}\right)
$$

for $1<\beta<4^{\frac{1}{p}}$.

To show that the constant

$$
\min \left[1, \max \left(2^{\frac{1}{p}}, \frac{4^{\frac{1}{p}}}{\beta}\right)\right]
$$

is the best possible in $X_{\beta}^{p}(1<p<\infty$ and $1<\beta<\infty)$, let us consider two sequences. We define the sequence $\left\{x_{n}\right\}$ by (see [4])

$$
x_{n}= \begin{cases}\frac{(2 k+1)^{2}-n}{4 k+1} e_{k}+e_{k+1} & \text { if }(2 k)^{2}<n \leq(2 k+1)^{2}, \\ e_{k+1}+\frac{n-(2 k+1)^{2}}{4 k+3} e_{k+2} & \text { if }(2 k+1)^{2}<n \leq(2 k+2)^{2},\end{cases}
$$


where $\left\{e_{k}\right\}$ is the standard basis in $l^{p}$. Then we have $x_{n} \rightarrow 0, x_{n+1}-x_{n} \rightarrow 0$,

$$
\operatorname{diam}_{a\|\cdot\|}\left\{x_{n}\right\}=\max \left(\frac{4^{\frac{1}{p}}}{\beta}, 1\right)
$$

and

$$
\left\|x_{(2 k+1)^{2}}\right\|=1 \text {. }
$$

This yields

$$
\begin{aligned}
& \max \left(\frac{4^{\frac{1}{p}}}{\beta}, 1\right) \cdot \inf _{x \in \operatorname{conv}_{\left\{x_{n}\right\}}\left(\liminf _{n}\left\|x_{n}-x\right\|\right)} \\
& =\max \left(\frac{4^{\frac{1}{p}}}{\beta}, 1\right) \cdot r_{a}\left(\left\{x_{(2 k+1)^{2}}\right\}\right)=\max \left(\frac{4^{\frac{1}{p}}}{\beta}, 1\right)=\operatorname{diam}_{a\|\cdot\|}\left(\left\{x_{n}\right\}\right) .
\end{aligned}
$$

The second sequence is defined as follows:

$$
x_{n}=\left[r_{n} \cos \left(\frac{n-(2 k)^{2}}{8 k+4} \cdot \frac{\pi}{2}\right)\right] e_{k}+\left[r_{n} \sin \left(\frac{n-(2 k)^{2}}{8 k+4} \cdot \frac{\pi}{2}\right)\right] e_{k+1},
$$

where

$$
r_{n}=\left\{\left[\cos \left(\frac{n-(2 k)^{2}}{8 k+4} \cdot \frac{\pi}{2}\right)\right]^{p}+\left[\sin \left(\frac{n-(2 k)^{2}}{8 k+4} \cdot \frac{\pi}{2}\right)\right]^{p}\right\}^{-\frac{1}{p}}
$$

for $(2 k)^{2}<n \leq(2 k+2)^{2}$ and $k=1,2, \ldots$ For this sequence we get $x_{n} \rightarrow 0$, $x_{n+1}-x_{n} \rightarrow 0$,

$$
\operatorname{diam}_{a\|\cdot\|}\left\{x_{n}\right\}=\max \left(1, \frac{2^{\frac{1}{p}}}{\beta}\right)
$$

and

$$
\left\|x_{4 k^{2}+4 k+2}\right\|=\max \left(\frac{1}{2^{\frac{1}{p}}}, \frac{1}{\beta}\right)=\frac{1}{2^{\frac{1}{p}}} \max \left(1, \frac{2^{\frac{1}{p}}}{\beta}\right) .
$$

Hence we obtain

$$
\begin{gathered}
2^{\frac{1}{p}} \cdot \inf _{x \in \operatorname{conv}\left\{x_{n}\right\}}\left(\liminf _{n}\left\|x_{n}-x\right\|\right)= \\
=2^{\frac{1}{p}} \cdot r_{a}\left(\left\{x_{4 k^{2}+4 k+2}\right\}\right)=\max \left(1, \frac{2^{\frac{1}{p}}}{\beta}\right)=\operatorname{diam}_{a\|\cdot\|}\left(\left\{x_{n}\right\}\right) .
\end{gathered}
$$

This completes the proof.

Remark 5.1. A Banach space $Y$ which is isomorphic to $X_{\beta}^{p}$ with $1<\beta<$ $4^{\frac{1}{p}}$ has the weak fixed point property for nonexpansive mappings if

$$
d\left(Y, X_{\beta}^{p}\right)<\min \left(2^{\frac{1}{p}}, \frac{4^{\frac{1}{p}}}{\beta}\right)
$$


(see Remark 2.3), but recently T. Domínguez Benavides and M.Á. Japón Pineda obtained a better result for $X_{\beta}^{2}$. Namely, if

$$
d\left(Y, X_{\beta}^{2}\right)<M\left(X_{\beta}^{2}\right)= \begin{cases}\sqrt{3} & \text { for } 1<\beta \leq \sqrt{\frac{3}{2}}, \\ \frac{\sqrt{2}}{\beta}\left(1+\sqrt{\frac{\beta^{2}-1}{2}}\right) & \text { for } \sqrt{\frac{3}{2}}<\beta<\sqrt{2}, \\ 1+\frac{1}{\sqrt{2}} & \text { for } \sqrt{2} \leq \beta,\end{cases}
$$

then $Y$ has the weak fixed point property [16]. But we have to mention that in the second part of our paper the coefficient $w$-SOC $(X)$ is applied to the problem of existence of fixed points of asymptotically regular uniformly lipschitzian semigroups, where till now we have not been able to use the coefficient $M(X)$.

\section{Comparison of the basic geometric coefficients of Banach SPACES}

As we mentioned in Section 2 the following inequalities

$$
A N(X) \leq w-A N(X)
$$

and

$$
W C S(X) \leq w-S O C(X)
$$

are always valid. The following examples show that for particular spaces strict inequalities may occur.

Example 6.1. If we take the Cartesian product $X_{\sqrt{2}}^{2} \times l^{1}$ equipped with the $l^{1}$ norm, then this space is nonreflexive and therefore by Theorem 2.1,

$A N\left(X_{\sqrt{2}}^{2} \times l^{1}\right)=1$, but after applying Theorems 2.2, 4.2 and 5.1 we obtain $w-A N\left(X_{\sqrt{2}}^{2} \times l^{1}\right)=w-S O C\left(X_{\sqrt{2}}^{2} \times l^{1}\right)=\sqrt{2}$.

Example 6.2. Taking $X_{\beta}^{2}$ with $1<\beta<\sqrt{2}$ and applying Theorem 5.1 we obtain

$$
1<W C S\left(X_{\beta}^{2}\right)=\frac{\sqrt{2}}{\beta}<w-S O C\left(X_{\beta}^{2}\right)=\sqrt{2} \text {. }
$$

Example 6.3. Let us consider the product space

$$
X=\left\{x=\left\{x_{p}\right\}_{p=1}^{\infty}: x_{p} \in l^{p},\left(\sum_{p=1}^{\infty}\left\|x_{p}\right\|_{p}^{2}\right)^{\frac{1}{2}}=\|x\|\right\} .
$$

Since $X$ contains isometric copies of $l^{p}$ for every $p$ and since both the semiOpial coefficient and the asymptotic normal structure coefficient satisfy

$$
w-S O C\left(l^{p}\right)=A N\left(l^{p}\right)=2^{\frac{1}{p}},
$$

the space $X$ has neither the uniform asymptotic normal structure nor the uniform semi-Opial properties. But it easy to observe that $X$ is still SO. In fact, it has the Opial property [26]. 
Acknowledgments. The third author was partially supported by the Fund for the Promotion of Research at the Technion. Part of the work on this paper was done when the third author visited the Institute of Mathematics at UMCS. He thanks the Institute and its members for their hospitality.

All the authors thank the referee for several useful comments and corrections.

\section{REFERENCES}

[1] A. G. Aksoy and M. A. Khamsi, Nonstandard methods in fixed point theory, SpringerVerlag, New York, 1990.

[2] J. M. Ayerbe Toledano, T. Domínguez Benavides and G. Lopez Acedo, Measures of noncompactness in metric fixed point theory, Birkhaüser Verlag, Basel, 1997.

[3] J. M. Ayerbe and H.-K. Xu, On certain geometric coefficients of Banach spaces related to fixed point theory, Panamer. Math. J. 3 (1993), 47-59.

[4] J.-B. Baillon and R. Schöneberg, Asymptotic normal structure and fixed points of nonexpansive mappings, Proc. Amer. Math. Soc. 81 (1981), 257-264.

[5] L. P. Belluce, W. A. Kirk and E. F. Steiner, Normal structure in Banach spaces, Pacific J. Math. 26 (1968), 433-440.

[6] M. S. Brodskii and D. P. Milman, On the center of a convex set, Dokl. Akad. Nauk SSSR 59 (1948), 837-840.

[7] M. Budzyńska, W. Kaczor and M. Koter-Mórgowska, Asymptotic normal structure, semi-Opial property and fixed points, Ann. Univ. Mariae Curie-Skłodowska Sect. A, 50 (1996), 33-41.

[8] M. Budzyńska, W. Kaczor, M. Koter-Mórgowska and T. Kuczumow, Asymptotic normal structure and semi-Opial property, Proc. Second World Congress of Nonlinear Analysts, Athens, Greece, 1996, Nonlinear Anal. 30 (1997), 3505-3515.

[9] W. l. Bynum, Normal structure coefficients for Banach spaces, Pacific J. Math. 86 (1980), 427-436.

[10] E. Casini and E. Maluta, Fixed points of uniformly lipschitzian mappings in spaces with uniformly normal structure, Nonlinear Anal. 9 (1985), 103-108.

[11] M. M. Day, Normed linear spaces, Springer-Verlag, Berlin, 1973.

[12] J. Diestel, Sequences and series in Banach spaces, Springer-Verlag, New York, 1984.

[13] T. Domínguez Benavides, Some properties of the set and ball measures of noncompactness and applications, J. London Math. Soc. 34 (1986), 120-128.

[14] T. Domínguez Benavides, Weak uniform normal structure in direct sum spaces, Studia Math. 103 (1992), 283-290.

[15] T. Domínguez Benavides, Fixed point theorems for uniformly lipschitzian mappings and asymptotically regular mappings, Nonlinear Anal., to appear.

[16] T. Domínguez Benavides and M.Á. Japón Pineda, Stability of the fixed point property for nonexpansive mappings in some classes of spaces, preprint.

[17] T. Domínguez Benavides and G. López Acedo, Lower bounds for normal structure coefficients, Proc. Roy. Soc. Edinburgh, 121 A (1992), 245-252.

[18] T. Domínguez Benavides, G. López Acedo and H.-K. Xu, Weak uniform normal structure and iterative fixed points of nonexpansive mappings, Colloq. Math. 68 (1995), $17-23$.

[19] K. Goebel and W. A. Kirk, Topics in metric fixed point theory, Cambridge University Press, Cambridge, 1990.

[20] K. Goebel and S. Reich, Uniform convexity, hyperbolic geometry, and nonexpansive mappings, Marcel Dekker, New York, 1984.

[21] L. A. Karlovitz, Some fixed point results for nonexpansive mappings, in Proc. Sem. Dalhousie Univ., Halifax, NS., 1975, Academic Press, New York, 1976, pp. 91-103. 
[22] L. A. Karlovitz, On nonexpansive mappings, Proc. Amer. Math. Soc. 55 (1976), 321325.

[23] L. A. Karlovitz, Existence of fixed points of nonexpansive mappings in a space without normal structure, Pacific J. Math. 66 (1976), 153-159.

[24] W. A. Kirk, A fixed theorem for mappings which do not increase distance, Amer. Math. Monthly, 72 (1965), 1004-1006.

[25] T. Kuczumow, S. Reich and M. Schmidt, A fixed point property of $l^{1}$-product spaces, Proc. Amer. Math. Soc. 119 (1993), 457-463.

[26] T. Kuczumow, S. Reich, M. Schmidt and A. Stachura, Strong asymptotic normal structure and fixed points in product spaces, Nonlinear Anal. 21 (1993), 501-515.

[27] T. C. Lim, On the normal structure coefficient and the bounded sequence coefficient, Proc. Amer. Math.Soc. 88 (1983), 262-264.

[28] P. K. Lin, Unconditional bases and fixed points of nonexpansive mappings, Pacific J. Math. 116 (1985), 69-76.

[29] E. Maluta, Uniformly normal structure and related coefficients, Pacific J. Math. 111 (1984), 357-369.

[30] S. A. Mariados and P. M. Soardi, A remark on asymptotic normal structure in Banach spaces, Rend. Sem. Mat. Univ. Politec. Torino 44 (1986), 393-395.

[31] D. P. Milman and V. D. Milman, The geometry of nested families with empty intersection - structure of the unit sphere of a nonreflexive space, Amer. Math. Soc. Transl., \#85, 1969, pp. 233-243.

[32] Z. Opial, Weak convergence of the sequence of successive approximations for nonexpansive mappings, Bull. Amer. Math. Soc. 73 (1967), 591-597.

[33] S. Prus, On Bynum's fixed point theorem, Atti Sem. Mat. Fis. Univ. Modena, 38 (1990), 535-545.

[34] B. Sims and M. Smyth, On non-uniform conditions giving weak structure, Questiones Mathematicae 18 (1995), 9-19.

[35] B. Sims and M. A. Smyth, On some Banach space properties sufficient for weak normal structure and their permanence properties, preprint.

[36] K.-K. Tan, A note on asymptotic normal structure and close-to-normal structure, Canad. Math. Bull. 25 (1982), 339-343.

[37] H.-K. Xu, Geometrical coefficients of Banach spaces and nonlinear mappings, preprint.

[38] X. T. Yu, A geometrically aberrant Banach space with uniformly normal structure, Bull. Aust. Math. Soc. 38 (1988), 99-103.

[39] W. Zhao, Geometrical coefficients and measures of noncompactness, Ph.D. Dissert., Univ Glasgow, 1992.

[40] G.-L. Zhang, Weakly convergent sequence coefficient of product space, Proc. Amer. Math. Soc. 117 (1993), 637-643.

Monika Budzyńska and Tadeusz Kuczumow

INSTYTUT MATEMATYKI UMCS

20-031 LuBLIN, POLAND

E-mail address: tadek@golem.umcs.lublin.pl

SiMEON REICH

Department of Mathematics

The Technion-Israel Institute of Technology 32000 HAIFA, ISRAEL

E-mail address: sreich@techunix.technion.ac.il 


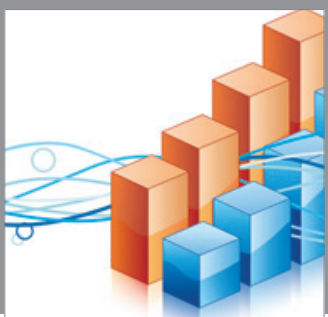

Advances in

Operations Research

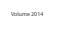

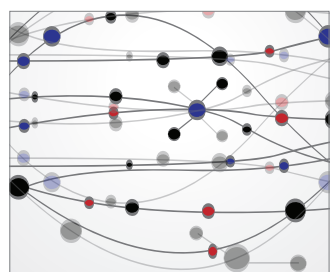

\section{The Scientific} World Journal
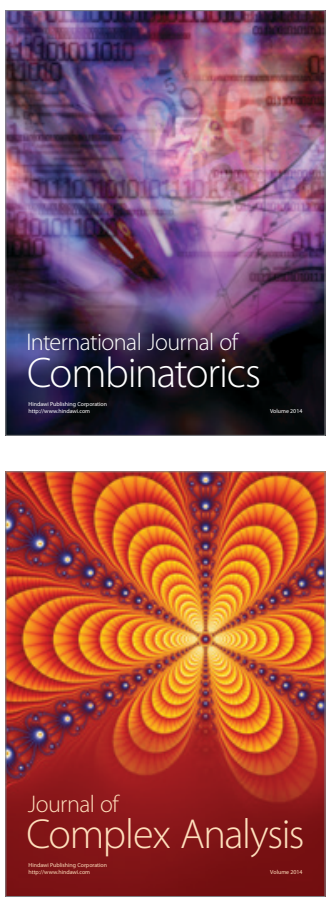

International Journal of

Mathematics and

Mathematical

Sciences
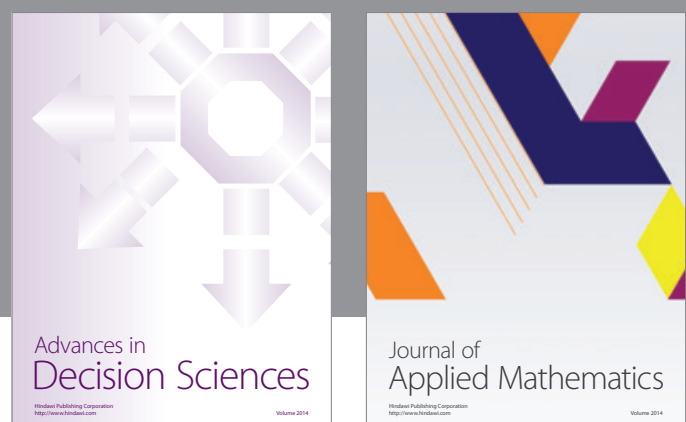

Journal of

Applied Mathematics
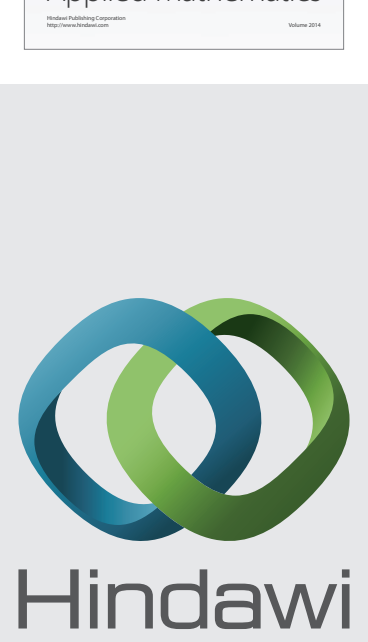

Submit your manuscripts at http://www.hindawi.com
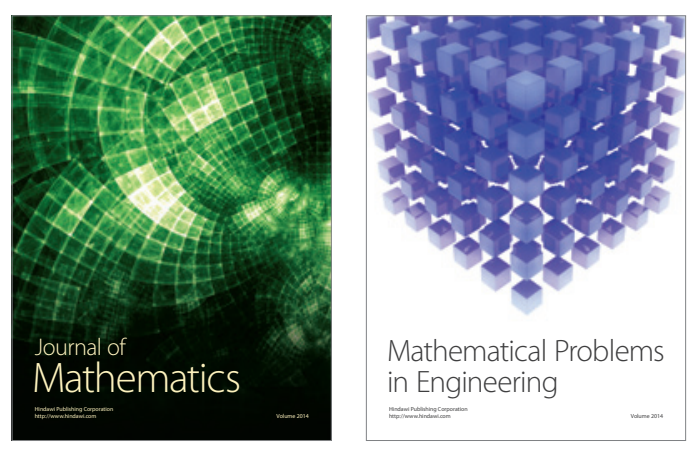

Mathematical Problems in Engineering
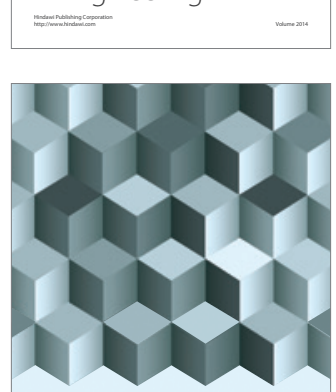

Journal of

Function Spaces
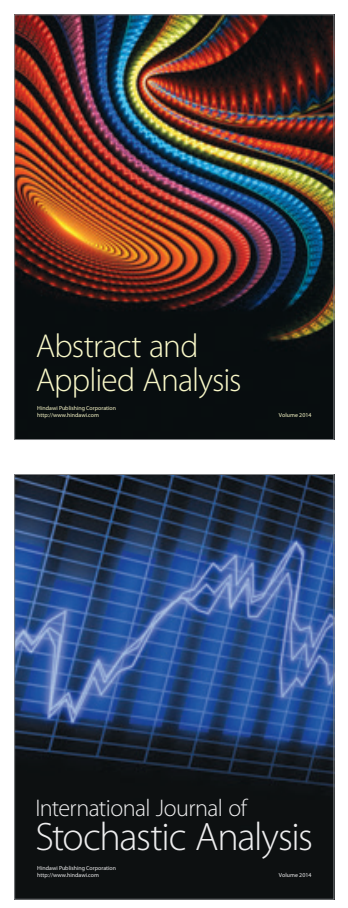

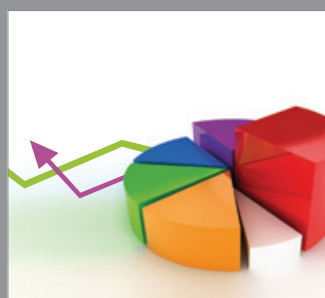

ournal of

Probability and Statistics

Promensencen
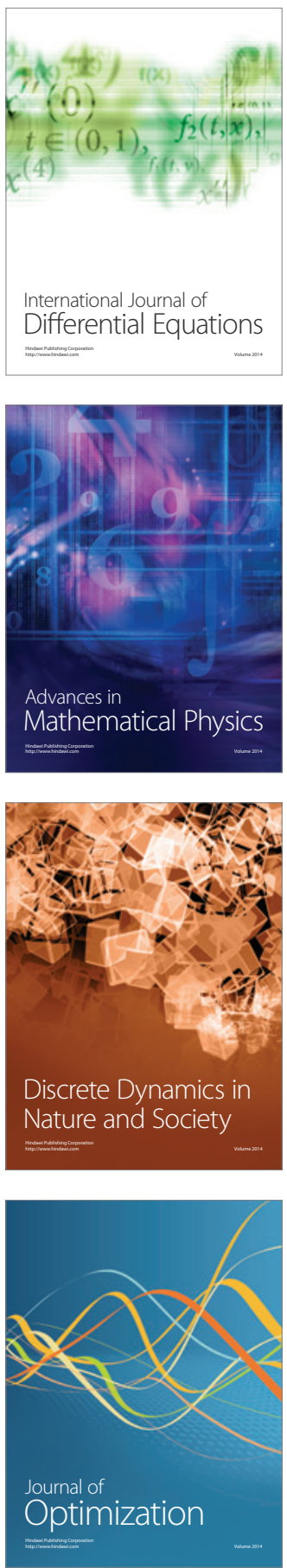